\title{
Efficacy and tolerability of ramucirumab monotherapy or in combination with paclitaxel in gastric cancer patients from the Expanded Access Program Cohort by the Korean Cancer Study Group (KCSG)
}

\author{
Minkyu Jung ${ }^{1} \cdot$ Min-Hee Ryu ${ }^{2} \cdot$ Do Youn $\mathrm{Oh}^{3} \cdot$ Myounghee $\mathrm{Kang}^{4} \cdot$ Dae Young Zang ${ }^{5} \cdot \ln \mathrm{Gyu}^{\mathrm{H}}$ wang ${ }^{6}$. \\ Keun-Wook Lee ${ }^{7} \cdot \mathrm{Ki} \mathrm{Hyang} \mathrm{Kim}^{8}$. Byoung Yong Shim ${ }^{9}$. Eun Kee Song ${ }^{10}$. Sun Jin Sym ${ }^{11}$. Hye Sook Han ${ }^{12}$. \\ Young Lee Park ${ }^{13}$. Jin Soo Kim ${ }^{14}$. Hyun Woo Lee ${ }^{15}$. Moon Hee Lee ${ }^{16}$. Dong-Hoe Koo ${ }^{17}$. Hong Suk Song ${ }^{18}$. \\ Namsu Lee ${ }^{19}$. Sung Hyun Yang ${ }^{20}$. Dae Ro Choi ${ }^{21}$. Young Seon Hong ${ }^{22}$. Kyoung Eun Lee ${ }^{23}$. Chi Hoon Maeng ${ }^{24}$. \\ Jin Ho Baek ${ }^{25}$. Samyong Kim ${ }^{26}$. Yeul Hong Kim ${ }^{27}$. Sun Young Rha ${ }^{1}$. Jae Yong Cho ${ }^{28}$. Yoon-Koo Kang ${ }^{2}$
}

Received: 25 September 2017 / Accepted: 30 January 2018 / Published online: 9 February 2018

(c) The International Gastric Cancer Association and The Japanese Gastric Cancer Association 2018

\begin{abstract}
Background Ramucirumab improves survival in gastric cancer patients. The efficacy and safety of ramucirumab outside of a clinical trial were evaluated using an expanded access program (EAP).

Methods Advanced gastric cancer patients treated with ramucirumab in combination with paclitaxel or with ramucirumab monotherapy in a Korean EAP were evaluated. Baseline characteristics were assessed for progression-free survival (PFS) and overall survival (OS), and adverse events were evaluated according to the treatment regimen.

Results Of 265 patients, 228 received ramucirumab plus paclitaxel, and 37 received ramucirumab monotherapy. Grade 3 or 4 neutropenia was more common with ramucirumab plus paclitaxel than with ramucirumab monotherapy (46.7 vs. $8.1 \%)$. Gastrointestinal (GI) perforation developed in seven patients (3.1\%) in the ramucirumab plus paclitaxel group. The overall response and disease control rates were 16.6 and $66.3 \%$ in the ramucirumab plus paclitaxel group, and 5.4 and $37.8 \%$ in the ramucirumab monotherapy group, respectively. PFS and OS were 3.8 and 8.6 months in the ramucirumab plus paclitaxel group, and 1.8 and 6.4 months in the ramucirumab monotherapy group, respectively. In multivariate analysis, alkaline phosphatase, albumin, and neutrophil-to-lymphocyte ratio (NLR) were the independent prognostic factors for PFS, while albumin, NLR, number of metastatic sites, and large amount of ascites were independent prognostic factors for OS.

Conclusion In the Korean EAP cohort, ramucirumab showed similar efficacy to the results of the previous trials for gastric cancer. However, the level of GI perforation was slightly increased in the ramucirumab plus paclitaxel group.
\end{abstract}

Keywords Gastric cancer · Ramucirumab - Second-line chemotherapy · Vascular endothelial growth factor · Expanded access program

Electronic supplementary material The online version of this article (https://doi.org/10.1007/s10120-018-0806-1) contains supplementary material, which is available to authorized users.

Minkyu Jung and Min-Hee Ryu have contributed equally to this work.

\section{Sun Young Rha \\ rha7655@yuhs.ac \\ Jae Yong Cho \\ chojy@yuhs.ac}

Extended author information available on the last page of the article

\section{Introduction}

Gastric cancer is a global health concern, with a high incidence and poor prognosis [1]. Surgical resection is the only curative treatment strategy for localized cancer and is used in combination with perioperative or adjuvant treatment $[2$, 3]. However, recurrence is common, and the prognosis is generally poor [4]. For advanced or metastatic gastric cancer patients, chemotherapy is the primary treatment [5].

Fluoropyrimidines plus platinum is the most common first-line chemotherapy for advanced gastric cancer [5, 6]. 
However, the median overall survival (OS) of patients with metastatic disease is approximately 1 year [7]. Recently, the second-line chemotherapy with irinotecan or docetaxel demonstrated increased OS compared with the best supportive care [8]. Paclitaxel as second-line chemotherapy also showed prolonged survival benefit and manageable toxicities [9].

Angiogenesis is strongly associated with tumor metastasis and progression. Vascular endothelial growth factor (VEGF) is a key regulator of angiogenesis, and several VEGF-targeting agents have been developed, including antibodies against VEGF and VEGF receptor (VEGFR), and tyrosine kinase inhibitors targeting VEGFR [10]. In gastric cancer, VEGF expression is also associated with poor prognosis [11]. Ramucirumab, a monoclonal antibody against VEGFR2, has shown a survival benefit when used as monotherapy or in combination with paclitaxel in patients with previously treated gastric or esophagogastric junction (EGJ) adenocarcinoma $[12,13]$.

However, few patients in Asia, where the incidence of gastric cancer is highest, have been enrolled in those trials $[12,13]$. In addition, the efficacy and safety of ramucirumab in a real-world setting have only been examined once outside of controlled clinical trials [14]. Recently, Fuchs et al. identified the prognostic factors for OS in gastric cancer from two-phase III studies of the second-line ramucirumab trials [15]. Therefore, we investigated the safety and efficacy of ramucirumab alone and in combination with paclitaxel and validated the prognostic factors by Fuchs in gastric cancer patients enrolled in an expanded access program (EAP) in Korea.

\section{Patients and methods}

\section{Patients}

Eligibility criteria included age $\geq 18$ years, histologically or cytologically confirmed gastric or EGJ adenocarcinoma, metastatic or unresectable disease, measurable or evaluable disease by abdominal and pelvic computed tomography, disease progression during treatment of firstline chemotherapy, and an Eastern Cooperative Oncology Group performance status (ECOG PS) score of 0 , 1 , or 2. Patients who met any of the following criteria were excluded: documented and/or symptomatic brain or leptomeningeal metastases, grade 3 or 4 gastrointestinal (GI) bleeding within 3 months prior to enrolment, arterial thromboembolic events, and uncontrolled or poorly controlled hypertension despite standard medical management.

\section{Treatment and data collection}

Patients received either $8 \mathrm{mg} / \mathrm{kg}$ ramucirumab monotherapy intravenously on days 1 and 15 , or the same dose in combination with $80 \mathrm{mg} / \mathrm{m}^{2}$ paclitaxel intravenously on days 1,8 , and 15 of a 28 day cycle. The decision to provide combination therapy or monotherapy was determined by the treating physicians. Patients received study treatment until disease progression, unacceptable toxicity, or withdrawal of consent. Age, sex, ECOG PS, presence of primary tumor, time to progression since prior therapy, tumor differentiation, metastatic sites, presence of large amount of ascites, and complete blood count with differential counts and serum parameters such as lactate dehydrogenase (LDH), alkaline phosphatase (ALP), aspartate aminotransferase (AST), albumin, sodium, and, creatinine were taken from patients' medical records. The large amount ascites was defined as ascites which needed paracentesis because of abdominal distension, early satiety, abdominal pain, or shortness of breath. Data on treatmentrelated adverse events were collected using the Common Terminology Criteria for Adverse Events version 4.0. We also collected data on adverse events of special interest related to ramucirumab (hemorrhage, bleeding, epistasis, proteinuria, thrombosis, and perforation), date of progression and death, and reason for cessation of ramucirumab. Radiological imaging was performed every 6 weeks. Responses were scored according to the Response Evaluation Criteria in Solid Tumors version 1.1.

\section{Statistical analysis}

Baseline pre-treatment white blood cell count, absolute neutrophil count, absolute lymphocyte count, neutrophil-to-lymphocyte ratio (NLR), and serum LDH level were analyzed. Progression-free survival (PFS) was defined as the time from the start of ramucirumab to disease progression or death from any cause. Patients without progression who were still alive at the time of analysis were censored. OS was defined as time from the start of ramucirumab to death from any cause. Univariate analysis for clinical and laboratory parameters was performed for OS and PFS. Survival curves of categorical variables were calculated using the Kaplan-Meier method and compared using the log-rank test. Multivariate analysis models of patient and tumor characteristics in association with PFS and OS were based on Cox-proportional hazards regression analyses. Two-tailed $P<0.05$ was considered statistically significant. Data were analysed using Statistical Package for Social Sciences (SPSS) software (version 23.0, Inc, Chicago, IL, USA). This EAP is registered at ClinicalTrials.gov (trial number NCT02065765). 
Table 1 Baseline characteristics of patients

\begin{tabular}{|c|c|c|c|c|c|}
\hline \multirow[t]{2}{*}{ Characteristics } & \multicolumn{2}{|c|}{$\begin{array}{l}\text { Ramucirumab plus pacli- } \\
\text { taxel }(n=228)\end{array}$} & \multicolumn{2}{|c|}{$\begin{array}{l}\text { Ramucirumab monotherapy } \\
(n=37)\end{array}$} & \multirow[t]{2}{*}{$P$ value } \\
\hline & Number & $\%$ & Number & $\%$ & \\
\hline Age & & & & & $0.007^{\mathrm{a}}$ \\
\hline Median & 57 & & 62 & & \\
\hline Range & $23-81$ & & $35-80$ & & \\
\hline Sex & & & & & $0.748^{\mathrm{b}}$ \\
\hline Male & 154 & 67.5 & 24 & 64.9 & \\
\hline Female & 74 & 32.5 & 13 & 35.1 & \\
\hline ECOG performance status & & & & & $0.708^{c}$ \\
\hline 0 & 51 & 22.4 & 7 & 18.9 & \\
\hline 1 & 170 & 74.5 & 28 & 75.7 & \\
\hline 2 & 7 & 3.1 & 2 & 5.4 & \\
\hline WHO classification & & & & & $0.135^{\mathrm{b}}$ \\
\hline Adenocarcinoma, well differentiated or moderately differentiated & 63 & 27.6 & 15 & 40.5 & \\
\hline Adenocarcinoma, poorly differentiated or signet ring cell & 141 & 61.9 & 16 & 43.2 & \\
\hline Others & 24 & 10.5 & 6 & 16.2 & \\
\hline Prior gastrectomy & & & & & $0.537^{\mathrm{b}}$ \\
\hline Yes & 107 & 46.9 & 22 & 59.5 & \\
\hline No & 121 & 53.1 & 15 & 40.5 & \\
\hline Previous treatment & & & & & $0.491^{\mathrm{c}}$ \\
\hline Triple: platinum and fluoropyrimidine with paclitaxel or irinotecan & 6 & 2.6 & 2 & 5.4 & \\
\hline Doublet: platinum and fluoropyrimidine & 169 & 74.1 & 30 & 81.1 & \\
\hline HER2 or other & 34 & 14.9 & 3 & 8.1 & \\
\hline Fluoropyrimidine monotherapy & 19 & 8.3 & 2 & 5.4 & \\
\hline Site of primary tumor & & & & & $0.396^{\mathrm{b}}$ \\
\hline EGJ & 30 & 13.2 & 7 & 18.9 & \\
\hline Gastric & 198 & 86.8 & 30 & 81.1 & \\
\hline HER2 status & & & & & $0.022^{\mathrm{c}}$ \\
\hline Positive & 32 & 14 & 0 & 0 & \\
\hline Negative & 189 & 82.9 & 34 & 91.9 & \\
\hline Unknown & 7 & 3.1 & 3 & 8.9 & \\
\hline Disease & & & & & $0.281^{\mathrm{b}}$ \\
\hline Measurable & 158 & 69.3 & 28 & 75.7 & \\
\hline Non-measurable & 70 & 30.7 & 9 & 24.3 & \\
\hline Number of metastatic sites & & & & & $0.991^{\mathrm{b}}$ \\
\hline $0-2$ & 166 & 72.8 & 27 & 73 & \\
\hline$\geq 3$ & 62 & 27.2 & 10 & 27 & \\
\hline Large amount of ascites ${ }^{\mathrm{d}}$ & & & & & $0.265^{\mathrm{b}}$ \\
\hline Yes & 60 & 26.3 & 13 & 35.1 & \\
\hline No & 168 & 73.7 & 24 & 64.9 & \\
\hline
\end{tabular}

ECOG PS Eastern Cooperative Oncology Group Performance Status, WHO World Health Organization, HER2 human epidermal growth factor receptor 2

${ }^{a}$ Analyzed using Kruskal-Wallis test

${ }^{\mathrm{b}}$ Analyzed using the $\chi^{2}$ test

${ }^{\mathrm{c}}$ Analyzed using the Mann-Whitney $U$ test

${ }^{\mathrm{d}}$ Need to paracentesis 
Table 2 Adverse events of ramucirumab plus paclitaxel and ramucirumab monotherapy

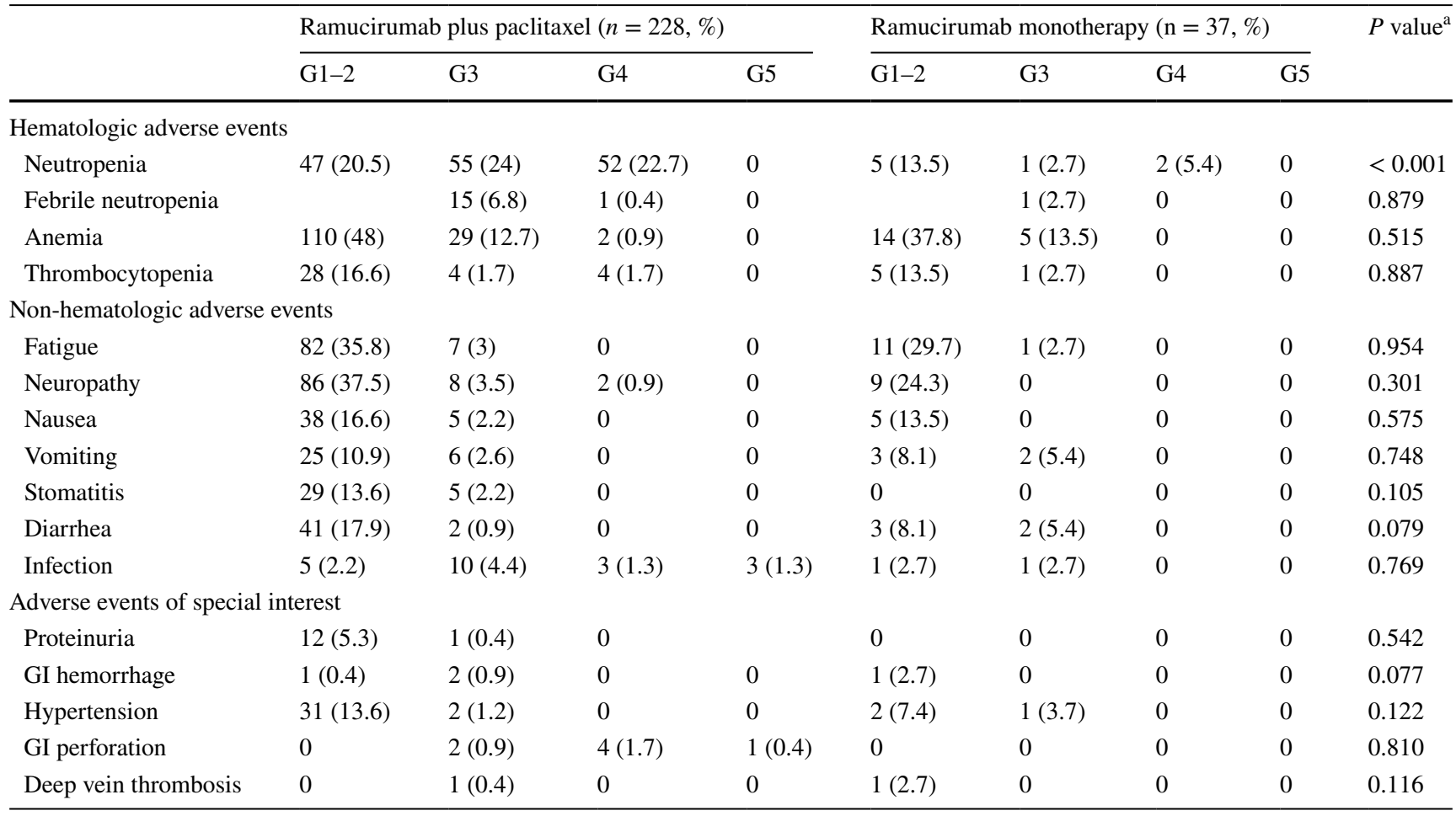

*Hypertension was evaluated in 169 and 27 patients in the ramucirumab plus paclitaxel group and the ramucirumab monotherapy group, respectively. GI gastrointestinal

a Analyzed using the Fisher's exact test
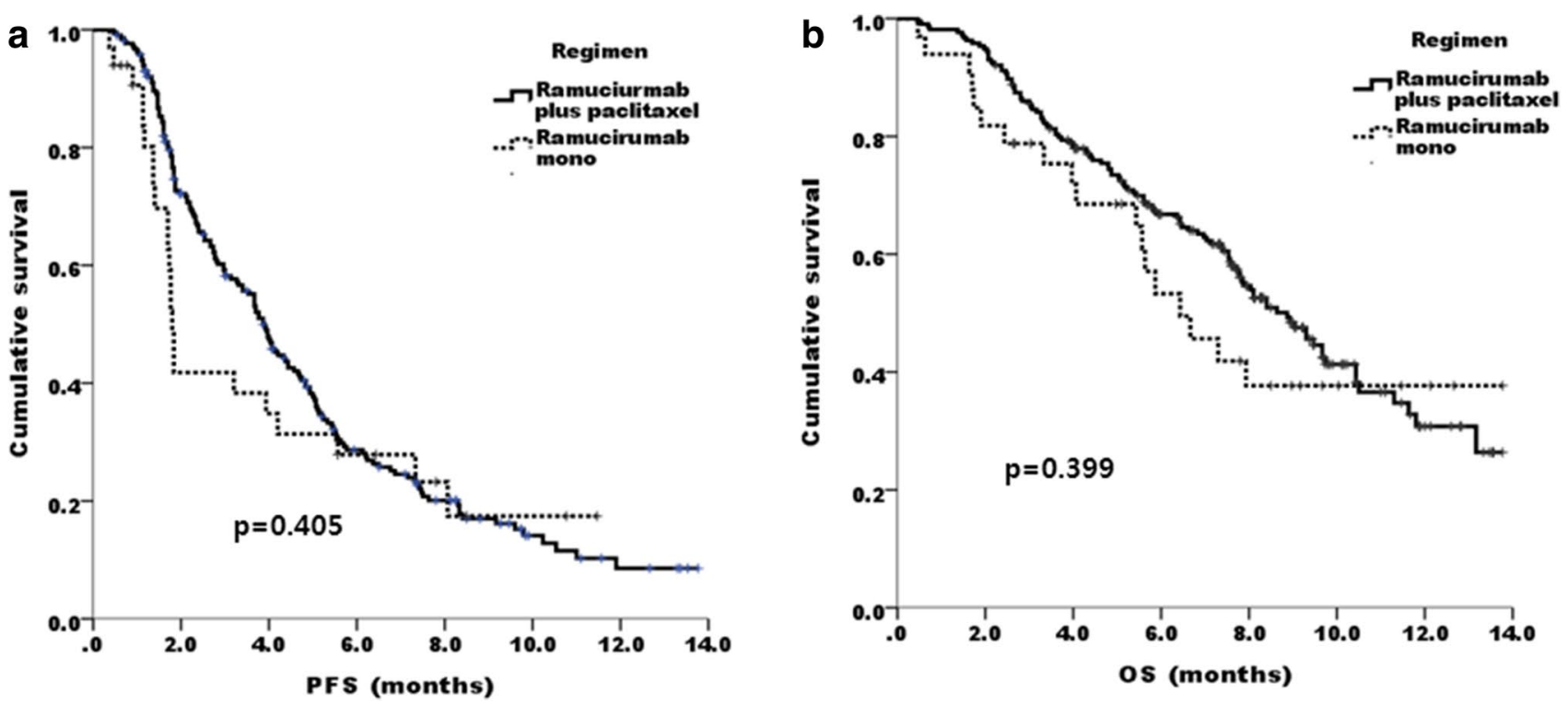

Fig. 1 Kaplan-Meier estimates of PFS a and OS b. PFS progression-free survival, OS overall survival 


\section{Results}

\section{Patient characteristics}

Between June 2015 and December 2015, data from 265 patients involved in a ramucirumab EAP were collected from 28 Korean hospitals. At the time of data cut-off (August 31, 2016), 228 patients $(86.4 \%)$ received combination therapy and 37 (13.7\%) received monotherapy. Twenty-five (9.4\%) were receiving treatment of the EAP. Patient characteristics are summarized in Table 1 . The median age was higher in the monotherapy group than in the combination group $(62$ vs. 57 years, $P=0.007$ ). Nine patients (seven in the combination and two in the monotherapy group) had an ECOG PS of 1 at the time of screening; however, their ECOG PS had increased to 2 on administration of study medication. There was no difference in ECOG PS between the groups. About $30 \%$ of patients had evaluable disease but no measurable lesions in each treatment group.

\section{Safety}

Although adverse events were more common in the combination group, this was not statistically significant (91.2\% [208/228] vs. 78.3\% [29/37], $P=0.568$ ), except for neutropenia (Table 2). The incidence of grade 3 or 4 neutropenia was significantly higher in the combination group than in the monotherapy group (46.7 vs. $8.1 \%, P<0.001$ ). Grade 3 or 4 adverse events of special interest were infrequent in both groups. However, seven patients (3.7\%) in the combination group experienced GI perforation. Three underwent stent insertion (two in the colon and one in the gastrojejunostomy anastomosis site) before ramucirumab treatment because of bowel obstruction, and all developed perforations around the stent. Bowel perforation was related to procedures in two patients (drainage procedure of ascites and embolization for GI bleeding). In the remaining two patients, bowel perforation occurred without any intervention. The median time to perforation after ramucirumab treatment was 6 (4-19) weeks (Supplementary Table 1).

\section{Efficacy}

The median follow-up in surviving patients was 9.2 (interquartile range [IQR] 4.0-9.0) months in the combination group and 9.6 (IQR 2.7-8.7) months in the monotherapy group. There were $174(76.3 \%)$ and $27(73 \%)$ cases of disease progression and $120(52.6 \%)$ and $22(59.5 \%)$ deaths in the combination and monotherapy group, respectively. Disease progression was the most common reason for treatment discontinuation (76.4 and $76.7 \%$ for combination and monotherapy, respectively), and the number of discontinuation due to adverse events were 12 (6.3\%) and 1 (3.3\%), respectively (Supplementary Table 2).

PFS $(3.8,95 \%$ confidence interval [CI], 3.4-4.4 months vs. $1.8,95 \%$ CI $1.7-1.9$ months) and OS (8.6, 95\% CI, 7.7-10.0 months vs. $6.4,95 \%$ CI $4.4-8.5$ months) were longer in the combination than in the monotherapy group (Fig. 1). The estimated 6 month PFS and OS rates were, respectively, 28.5 and $66.9 \%$ in the combination group, and 27.7 and $53.2 \%$ in the monotherapy group. The proportion of patients who achieved an objective response was higher in the combination group than in the monotherapy group (16.6 vs. 5.4\%, $P=0.053)$. The disease control rate (DCR) was also significantly higher in the combination group (66.3 vs. $37.8 \%$, $P=0.001$ ) (Table 3 ). In addition, the overall response was not different according to NLR status ( 16.1 vs. $17.5 \%, P=0.828$ ), but disease control rate was higher in low NLR group than high one (69.9 vs. $52.5 \%, P=0.032)$.

\section{Factors related to survival}

By univariate analysis, time to progression since prior therapy $(<6$ months), low sodium $(<135 \mathrm{mmol} / \mathrm{L})$, high ALP ( $>150 \mathrm{U} / \mathrm{L})$, low albumin (3.5 U/L), high LDH ( $\geq 247 \mathrm{IU} / \mathrm{L})$, low lymphocyte $(<1500 / \mu \mathrm{L})$, high NLR $(\geq 5)$, and the number of metastatic sites ( $\geq 3$ ) were related to poor PFS (Table 4), and, time to progression since prior therapy ( $<6$ months), presence of a primary tumor, poor ECOG PS (1 and 2), low sodium $(<135 \mathrm{mmol} / \mathrm{L})$, high ALP $(>150 \mathrm{U} / \mathrm{L})$, low albumin (3.5 U/L), high LDH ( $\geq 247$ IU/L), high neutrophil ( $>7500 /$ $\mu \mathrm{L})$, low lymphocyte $(<1500 / \mu \mathrm{L})$, high NLR, increased number of metastatic sites $(\geq 3)$, and a large amount of ascites were related to poor OS (Table 5). In multivariate analyses including each parameter that had a significant relationship with PFS and OS in univariate analysis, high ALP, low albumin, and high NLR were the independent factor for poor PFS, and

Table 3 Best overall response to ramucirumab plus paclitaxel and ramucirumab monotherapy

\begin{tabular}{|c|c|c|c|c|c|}
\hline & \multicolumn{2}{|c|}{$\begin{array}{l}\text { Ramucirumab } \\
\text { plus paclitaxel } \\
(n=228)\end{array}$} & \multicolumn{2}{|c|}{$\begin{array}{l}\text { Ramucirumab } \\
\text { monotherapy } \\
(n=37)\end{array}$} & \multirow[t]{2}{*}{$P$ value $^{\mathrm{a}}$} \\
\hline & Number & $\%$ & Number & $\%$ & \\
\hline Complete response & 1 & 0.4 & 0 & 0 & 0.021 \\
\hline Partial response & 37 & 16.2 & 2 & 5.4 & \\
\hline Stable disease & 113 & 49.6 & 12 & 32.4 & \\
\hline Progressive disease & 63 & 27.6 & 17 & 45.9 & \\
\hline $\begin{array}{l}\text { Not evaluable or not } \\
\text { assessed }\end{array}$ & 14 & 6.2 & 6 & 16.3 & \\
\hline Overall response rate & 38 & 16.6 & 2 & 5.4 & 0.127 \\
\hline Disease control rate & 151 & 66.3 & 14 & 37.8 & 0.008 \\
\hline
\end{tabular}

\footnotetext{
${ }^{a}$ Analyzed using the Fisher's exact test
} 
Table 4 Univariate and multivariate analyses for progression-free survival

\begin{tabular}{|c|c|c|c|c|c|}
\hline & \multicolumn{3}{|c|}{ Univariate analysis } & \multicolumn{2}{|c|}{$\begin{array}{l}\text { Multivariate } \\
\text { analysis }\end{array}$} \\
\hline & $\begin{array}{l}\text { Median } \\
\text { (months) }\end{array}$ & $95 \% \mathrm{CI}$ & $P$ value $^{\mathrm{a}}$ & $\overline{\mathrm{HR}}$ & $P$ value \\
\hline Sex & & & 0.064 & & \\
\hline Male & 4 & $3.4-4.6$ & & & \\
\hline Female & 2.3 & $1.9-2.7$ & & & \\
\hline Age, years & & & 0.332 & & \\
\hline$\geq 65$ & 2.5 & $1.8-3.3$ & & & \\
\hline$<65$ & 3.8 & $3.3-4.2$ & & & \\
\hline Time to progression since prior therapy & & & 0.013 & & 0.140 \\
\hline$<6$ months & 2.8 & $1.8-3.7$ & & 1.335 & \\
\hline$\geq 6$ months & 4.7 & $3.8-5.7$ & & 1 & \\
\hline HER2 & & & 0.605 & & \\
\hline Negative & 3.7 & $3.1-4.3$ & & & \\
\hline Positive & 3.7 & $2.2-5.2$ & & & \\
\hline Presence of a primary tumor & & & 0.094 & & \\
\hline Yes & 3.7 & $2.8-4.6$ & & & \\
\hline No & 3.8 & $2.4-5.3$ & & & \\
\hline Histology & & & 0.289 & & \\
\hline Well/moderate differentiated & 4.3 & $3.4-5.2$ & & & \\
\hline Poor/singnet ring cell/mixed & 3.1 & $2.4-3.8$ & & & \\
\hline ECOG PS & & & 0.119 & & \\
\hline 0 & 4.9 & $4.2-5.7$ & & & \\
\hline 1 & 3.3 & $2.5-4.0$ & & & \\
\hline 2 & 1.8 & $1.6-2.0$ & & & \\
\hline Sodium, mmol/L & & & 0.026 & & 0.623 \\
\hline$<135$ & 2.3 & $1.9-2.8$ & & 1.114 & \\
\hline$\geq 135$ & 3.9 & $3.4-4.3$ & & 1 & \\
\hline ALP, U/L & & & 0.008 & & 0.018 \\
\hline$\leq 150$ & 4.1 & $3.3-4.7$ & & 1 & \\
\hline$>150$ & 2.7 & $2.0-3.3$ & & 1.643 & \\
\hline Albumin, U/L & & & $<0.001$ & & 0.003 \\
\hline$<3.5$ & 2.5 & $1.7-3.3$ & & 1.849 & \\
\hline$\geq 3.5$ & 4.1 & $3.6-4.7$ & & 1 & \\
\hline AST, U/L & & & 0.330 & & \\
\hline$<35$ & 3.7 & $3.1-4.3$ & & & \\
\hline$\geq 35$ & 3.1 & $1.6-4.6$ & & & \\
\hline $\mathrm{LDH}, \mathrm{U} / \mathrm{L}(n=133)$ & & & 0.044 & & 0.227 \\
\hline$<247$ & 4 & $2.6-5.4$ & & 1 & \\
\hline$\geq 247$ & 3.1 & $2.1-4.2$ & & 1.268 & \\
\hline Neutrophil/ $\mu \mathrm{L}$ & & & 0.068 & & \\
\hline$<7500$ & 3.8 & $3.2-4.3$ & & & \\
\hline$\geq 7500$ & 2.7 & $1.5-3.8$ & & & \\
\hline Lymphocyte/ $\mu \mathrm{L}$ & & & 0.002 & & \\
\hline$<1500$ & 2.5 & $2.0-3.1$ & & & \\
\hline$\geq 1500$ & 4.4 & $3.8-5.1$ & & & \\
\hline NLR & & & 0.003 & & 0.006 \\
\hline$<5$ & 4 & $3.5-4.6$ & & 1 & \\
\hline$\geq 5$ & 2.3 & $1.4-3.3$ & & 2.014 & \\
\hline Number of metastatic sites & & & 0.027 & & 0.753 \\
\hline $0-2$ & 3.9 & $3.2-4.6$ & & 1 & \\
\hline
\end{tabular}


Table 4 (continued)

\begin{tabular}{|c|c|c|c|c|c|}
\hline & \multicolumn{3}{|c|}{ Univariate analysis } & \multicolumn{2}{|c|}{$\begin{array}{l}\text { Multivariate } \\
\text { analysis }\end{array}$} \\
\hline & $\begin{array}{l}\text { Median } \\
\text { (months) }\end{array}$ & $95 \% \mathrm{CI}$ & $P$ value $^{\mathrm{a}}$ & $\overline{\mathrm{HR}}$ & $P$ value $^{\mathrm{b}}$ \\
\hline$\geq 3$ & 2.7 & $1.6-3.8$ & & 1.075 & \\
\hline Peritoneal metastases & & & 0.773 & & \\
\hline No & 3.9 & $3.2-4.6$ & & & \\
\hline Yes & 3.3 & $2.4-4.1$ & & & \\
\hline Large amount of Ascites ${ }^{\mathrm{c}}$ & & & 0.115 & & \\
\hline No & 3.8 & $3.3-4.4$ & & & \\
\hline Yes & 3.1 & $1.8-4.5$ & & & \\
\hline Regimen & & & 0.112 & & \\
\hline Ramucirumab plus paclitaxel & 3.8 & $3.4-4.4$ & & & \\
\hline Ramucirumab & 1.8 & $1.7-1.9$ & & & \\
\hline
\end{tabular}

ECOG PS Eastern Cooperative Oncology Group Performance Status WHO World Health Organization, $H E R 2$ human epidermal growth factor receptor 2, $A L P$ alkaline phosphatase, $L D H$ lactate dehydrogenase, $A S T$ aspartate aminotransferase, $N R$ not reached, NLR: neutrophil-lymphocyte ratio

${ }^{\mathrm{a}}$ Analyzed using log-rank test

${ }^{\mathrm{b}}$ Analyzed using Cox-regression model

${ }^{\mathrm{c}}$ Need to paracentesis

low albumin, high NLR, increased number of metastatic sites $(\geq 3)$, and large amount of ascites were independent factors for poor OS (Tables 4, 5). The PFS and OS of patients with a low NLR were significantly longer than those of patients with a high NLR (median PFS, 4.2 vs. 2.7 months, $P=0.004$; median OS, 9.0 vs. 6.4 months, $P=0.001$ ) (Fig. 2). HER2 status did not affect PFS or OS. In the event of progression after ramucirumab treatment, the proportion of patients treated with systemic chemotherapy was similar in both groups (56.9 vs. $51.9 \%, P=0.806)$, and the most common chemotherapeutic agent was irinotecan.

\section{Discussion}

In patients with advanced gastric cancer who were treated with prior chemotherapy, ramucirumab has shown improved survival when administered in combination with paclitaxel or as monotherapy [12, 13], but this has not been extensively studied outside of clinical trials [14]. To our knowledge, this study is the first to report outcomes of ramucirumab in a large cohort in a real-world clinical setting in Asia. In addition, we validated the prognostic factors by Fuchs [15] and investigated predictive candidate biomarkers for the efficacy of ramucirumab in our EAP data.

In our data, ramucirumab showed similar efficacy and toxicities to those found in the RAINBOW and REGARD trials $[12,13]$. In the combination group, the ORR and DCR were slightly lower than those in RAINBOW (ORR, 16.7 vs.
28\%; DCR, 66.2 vs. 79\%) [13]. The safety profile of combination therapy in our study was similar to the RAINBOW result (Supplementary Table 3), including hematological and non-hematological adverse events and VEGFR pathwayrelated toxicities. In general, ramucirumab monotherapy did not developed severe neutropenia and febrile neutropenia [12]. However, in our study, febrile neutropenia (2.7\%) and severe neutropenia $(8.1 \%)$ were developed in patients treated with ramucirumab monotherapy. This patient who developed febrile neutropenia was suffered from upper respiratory symptom, such as cough and rhinorrhoea, at the time of admission for febrile neutropenia. The subject's condition was improved shortly after supportive care. Although the febrile neutropenia was possibly related to severe respiratory syndrome, the investigator could not exclude the causal relationship between febrile neutropenia and ramucirumab. Three patients with severe neutropenia had grade 1 neutropenia at baseline. Therefore, the neutropenia from ramucirumab monotherapy might be developed in patients with bone marrow suppression before the treatment.

GI perforation rate was slightly higher than RAINBOW trial [7 (3.1\%) of 228 vs. 4 (1.2\%) of 327]. In five of seven patients, GI perforation was associated with procedures, most commonly stent therapy (3 out of 7 ). According to a meta-analysis regarding perforation in colorectal stenting, bevacizumab-based therapy was identified as a risk factor, with a perforation rate of $12.5 \%$ [16]. In contrast, the perforation risk was $7.0 \%$ in patients treated with chemotherapy without bevacizumab and was $9 \%$ in patients not treated with chemotherapy during stent therapy [16]. Although a causal 
Table 5 Univariate and multivariate analyses for overall survival

\begin{tabular}{|c|c|c|c|c|c|}
\hline & \multicolumn{3}{|c|}{ Univariate analysis } & \multicolumn{2}{|c|}{$\begin{array}{l}\text { Multivariate } \\
\text { analysis }\end{array}$} \\
\hline & $\begin{array}{l}\text { Median } \\
\text { (months) }\end{array}$ & $95 \% \mathrm{CI}$ & $P$ value $^{\mathrm{a}}$ & HR & $P$ value $^{\mathrm{b}}$ \\
\hline Sex & & & 0.076 & & \\
\hline Male & 8.9 & $7.6-10.1$ & & & \\
\hline Female & 7.3 & $5.0-9.6$ & & & \\
\hline Age, years & & & 0.534 & & \\
\hline$\geq 65$ & 7.9 & $6.6-9.3$ & & & \\
\hline$<65$ & 8.4 & $7.1-9.6$ & & & \\
\hline Time to progression since prior therapy & & & 0.006 & & 0.228 \\
\hline$<6$ months & 7.6 & $6.5-8.7$ & & 1.383 & \\
\hline$\geq 6$ months & 9.7 & $8.0-11.5$ & & 1 & \\
\hline HER2 & & & 0.321 & & \\
\hline Negative & 8.1 & $7.2-9.0$ & & & \\
\hline Positive & 11.6 & $3.2-20.0$ & & & \\
\hline Presence of a primary tumor & & & 0.032 & & 0.815 \\
\hline Yes & 9.3 & $7.4-11.2$ & & 1.062 & \\
\hline No & 7.6 & $6.2-9.0$ & & 1 & \\
\hline Histology & & & 0.148 & & \\
\hline Well/moderate differentiated & 9.3 & $7.7-11.9$ & & & \\
\hline Poor/singnet ring cell/mixed & 7.8 & $6.4-9.2$ & & & \\
\hline ECOG PS & & & 0.001 & & 0.157 \\
\hline 0 & NR & & & 1 & \\
\hline 1 & 7.7 & $6.8-8.6$ & & 1.628 & 0.097 \\
\hline 2 & 5.2 & $1.5-8.8$ & & 2.35 & 0.109 \\
\hline Sodium, $\mathrm{mmol} / \mathrm{L}$ & & & $<0.001$ & & 0.794 \\
\hline$<135$ & 4.6 & $3.4-5.9$ & & 1.104 & \\
\hline$\geq 135$ & 9.0 & $8.0-10.1$ & & 1 & \\
\hline ALP, U/L & & & 0.015 & & 0.291 \\
\hline$\leq 150$ & 8.9 & $7.8-10.0$ & & 1 & \\
\hline$>150$ & 6.4 & $4.5-8.4$ & & 1.134 & \\
\hline Albumin, U/L & & & $<0.001$ & & 0.012 \\
\hline$<3.5$ & 5.2 & $3.9-6.4$ & & 1 & \\
\hline$\geq 3.5$ & 9.7 & $8.4-11.0$ & & 1.971 & \\
\hline AST, U/L & & & 0.833 & & \\
\hline$<35$ & 8.2 & $7.1-9.4$ & & & \\
\hline$\geq 35$ & 8.0 & $7.0-9.1$ & & & \\
\hline $\mathrm{LDH}, \mathrm{U} / \mathrm{L}(n=133)$ & & & 0.37 & & \\
\hline$<247$ & 8.9 & $7.0-10.8$ & & & \\
\hline$\geq 247$ & 8.7 & $6.8-11.5$ & & & \\
\hline Neutrophil/ $\mu \mathrm{L}$ & & & 0.005 & & \\
\hline$<7500$ & 8.6 & $7.6-9.7$ & & & \\
\hline$\geq 7500$ & 4.9 & $1.7-8.0$ & & & \\
\hline Lymphocyte/ $\mu \mathrm{L}$ & & & $<0.001$ & & \\
\hline$<1500$ & 6.4 & $5.2-7.5$ & & & \\
\hline$\geq 1500$ & 10.8 & $8.9-14.5$ & & & \\
\hline NLR & & & 0.001 & & 0.015 \\
\hline$<5$ & 9.3 & $8.3-10.3$ & & 1 & \\
\hline$V \geq 5$ & 4.1 & $2.2-6.0$ & & 2.269 & \\
\hline Number of metastatic sites & & & 0.008 & & 0.024 \\
\hline $0-2$ & 5.8 & $4.4-7.8$ & & 1 & \\
\hline
\end{tabular}


Table 5 (continued)

\begin{tabular}{|c|c|c|c|c|c|}
\hline & \multicolumn{3}{|c|}{ Univariate analysis } & \multicolumn{2}{|c|}{$\begin{array}{l}\text { Multivariate } \\
\text { analysis }\end{array}$} \\
\hline & $\begin{array}{l}\text { Median } \\
\text { (months) }\end{array}$ & $95 \% \mathrm{CI}$ & $P$ value $^{a}$ & $\overline{\mathrm{HR}}$ & $P$ value $^{\mathrm{b}}$ \\
\hline$\geq 3$ & 8.9 & $7.8-10.1$ & & 2.084 & \\
\hline Peritoneal metastases & & & 0.104 & & \\
\hline No & 9.0 & $7.4-10.6$ & & & \\
\hline Yes & 7.5 & $5.8-9.3$ & & & \\
\hline Large amount of Ascites ${ }^{c}$ & & & 0.001 & & 0.002 \\
\hline No & 9.3 & $7.9-10.7$ & & 1 & \\
\hline Yes & 5.8 & $4.1-7.6$ & & 2.511 & \\
\hline Regimen & & & 0.283 & & \\
\hline Ramucirumab plus paclitaxel & 8.6 & $7.6-9.7$ & & & \\
\hline Ramucirumab & 6.4 & $7.3-9.2$ & & & \\
\hline
\end{tabular}

ECOG PS Eastern Cooperative Oncology Group Performance Status WHO World Health Organization, $H E R 2$ human epidermal growth factor receptor 2, $A L P$ alkaline phosphatase, $L D H$ lactate dehydrogenase, $A S T$ aspartate aminotransferase, $N R$ not reached, NLR: neutrophil-lymphocyte ratio

${ }^{\mathrm{a}}$ Analyzed using log-rank test

${ }^{\mathrm{b}}$ Analyzed using cox-regression model

${ }^{\mathrm{c}}$ Need to paracentesis

relationship between GI perforation and stents in RAINBOW was not proven, the higher incidence of GI perforation in our results might be associated with GI stents. However, the incidences of other ramucirumab-related adverse events of special interest were similar to those observed in RAINBOW. Therefore, clinicians should be aware of the risk of
GI perforation in patients receiving combination treatment, especially those with GI stents.

Although the sample size was small, the ORR was slightly higher and the DCR slightly lower in the monotherapy group than in REGARD (ORR, 5.4 vs. 3\%; DCR, 37.8 vs. 49\%) [12]. The median OS for the monotherapy group was 6.4 months (IQR 7.3-9.2), which was slightly
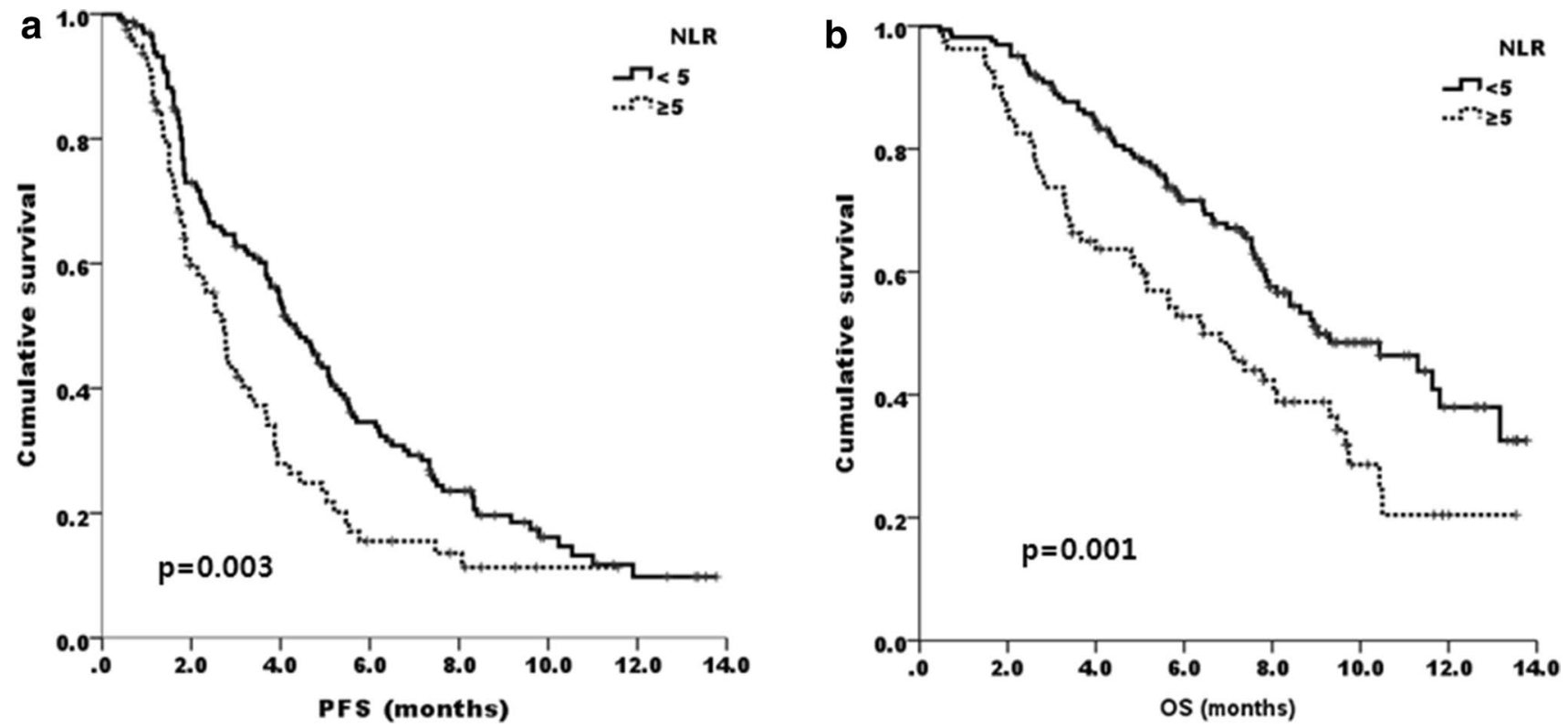

Fig. 2 Kaplan-Meier estimates of PFS a and OS b according to NLR. PFS progression-free survival, $O S$ overall survival, NLR neutrophil-tolymphocyte ratio 
longer than that in REGARD (median OS, 5.2 months, IQR 2.3-9.9) [12]. We had no treatment-related deaths or withdrawals due to drug-related adverse events from monotherapy. The most common adverse event in the monotherapy group was anemia (51.3\%); otherwise, the safety profile of monotherapy in our study was similar to that of REGARD (Supplementary Table 4) [12].

We included nine patients who had an ECOG PS of 2 during ramucirumab administration, although they had an ECOG PS $\leq 1$ at screening. Our results may be more representative of advanced gastric cancer patients in the realworld clinical setting, because our population included those who would have been excluded from a clinical trial because of poor medical condition.

Identifying predictive biomarkers to stratify patients for treatment is vital. Although several predictive biomarkers of anti-angiogenic therapy have been evaluated, no reproducible blood or tissue biomarkers have been identified [17]. In addition, none of the biomarkers, including VEGFR2 and HER2 in tumor tissue and soluble VEGF-C, -D, VEGFR1, and VEGFR3, was significantly associated with ramucirumab efficacy in REGARD [18]. In addition, Fuchs et al. identified 12 independent prognostic factors of poor survival from RAINBOW and REGARD trials [15]. The 12 prognostic factors of poor OS were peritoneal metastases, ECOG PS 1 , the presence of a primary tumor, time to progression since prior therapy $<6$ months, poor/unknown tumor differentiation, abnormally low blood levels of albumin, sodium, and/ or lymphocytes, abnormally high blood levels of neutrophils, AST, ALP, and/or LDH. Among the 12 prognostic factors, low albumin was the only independent prognostic factor of poor OS in our EAP data. Although Fuchs did not identify the prognostic marker for PFS, we applied the 12 prognostic markers to PFS in our study. Th low albumin and high ALP were independently associated with poor PFS in our study.

To discover another prognostic biomarker for PFS and OS, we evaluated baseline clinicopathologic parameters including blood laboratory test. In multivariate analysis, the high ALP, low Albumin, and high NLR were the independent factors for poor PFS, and low albumin, high NLR, number of metastatic sites, and large amount of ascites were independent factors for poor OS in our study. The independent prognostic markers for PFS are usually not identical to those for OS [19-21]. One explanation for the difference between prognostic factors of PFS and OS is that OS might be influenced by subsequent treatment after disease progression. In our study, 119 of $265(44.9 \%)$ patients were treated with subsequent treatment after progression with ramucirumab.

In conclusion, analysis of the current EAP cohort of Korean patients with advanced gastric cancer showed that ramucirumab treatment had a manageable safety profile and antitumor activity. These findings are consistent with the safety and activity profiles in the RAINBOW and REGARD trials. We observed that GI perforation occurred more frequently in patients with stents in the combination group; therefore, ramucirumab should be administered with caution in these patients.

Acknowledgements The EAP was sponsored by Lilly Company. We would like to thank the patients and investigators who participated in the Korean Ramucirumab EAP. This research was supported by the grant from the National R\&D Program for Cancer Control, Ministry of Health and Welfare, Republic of Korea (Sun Young Rha, 1520190). The funders had no role in the study design, data collection and analysis, decision to publish, or preparation of the manuscript.

\section{Compliance with ethical standards}

Conflict of interest The authors have declared no conflicts of interest.

Human rights statement and informed consent Each centre's institutional review board or independent ethics committee approved the study. This program followed the guiding principles of the Declaration of Helsinki and the Good Clinical Practice Guidelines of the International Conference on Harmonization. All patients provided written informed consent.

\section{References}

1. Torre LA, Bray F, Siegel RL, Ferlay J, Lortet Tieulent J, Jemal A. Global cancer statistics, 2012. Cancer J Clin. 2015;65:87-108.

2. Sakuramoto S, Sasako M, Yamaguchi T, Kinoshita T, Fujii M, Nashimoto A, et al. Adjuvant chemotherapy for gastric cancer with S-1, an oral fluoropyrimidine. N Engl J Med. 2007;357:1810-20.

3. Bang Y, Kim Y, Yang H, Chung HC, Park Y, Lee KH, et al. Adjuvant capecitabine and oxaliplatin for gastric cancer after D2 gastrectomy (CLASSIC): a phase 3 open-label, randomised controlled trial. Lancet. 2012;379:315-21.

4. Leung WK, Wu M, Kakugawa Y, Kim J, Yeoh K, Goh KL, et al. Screening for gastric cancer in Asia: current evidence and practice. Lancet Oncol. 2008;9:279-87.

5. Van Cutsem E, Moiseyenko VM, Tjulandin S, Majlis A, Constenla $\mathrm{M}$, Boni C, et al. Phase III study of docetaxel and cisplatin plus fluorouracil compared with cisplatin and fluorouracil as first-line therapy for advanced gastric cancer: a report of the V325 Study Group. J Clin Oncol. 2006;24:4991-7.

6. Cunningham D, Starling N, Rao S, Iveson T, Nicolson M, Coxon $\mathrm{F}$, et al. Capecitabine and oxaliplatin for advanced esophagogastric cancer. N Engl J Med. 2008;358:36-46.

7. Lordick F, Allum W, Carneiro F, Mitry E, Tabernero J, Tan P, et al. Unmet needs and challenges in gastric cancer: the way forward. Cancer Treat Rev. 2014;40:692-700.

8. Kang JH, Lim DH, Park K, Oh SY, Kwon H, Hwang IG, et al. Salvage chemotherapy for pretreated gastric cancer: a randomized phase III trial comparing chemotherapy plus best supportive care with best supportive care alone. J Clin Oncol. 2012;30:1513-8.

9. Hironaka S, Ueda S, Yasui H, Nishina T, Tsuda M, Tsumura T, et al. Randomized, open-label, phase III study comparing irinotecan with paclitaxel in patients with advanced gastric cancer without severe peritoneal metastasis after failure of prior combination chemotherapy using fluoropyrimidine plus platinum: WJOG 4007 trial. J Clin Oncol. 2013;31:4438-44. 
10. Goel HL, Mercurio AM. VEGF targets the tumour cell. Nat Rev Cancer. 2013;13:871-82.

11. Jüttner S, Wissmann C, Jöns T, Vieth M, Hertel J, Gretschel $\mathrm{S}$, et al. Vascular endothelial growth factor-D and its receptor VEGFR-3: two novel independent prognostic markers in gastric adenocarcinoma. J Clin Oncol. 2006;24:228-40.

12. Fuchs CS, Tomasek J, Yong CJ, Dumitru F, Passalacqua R, Goswami C, et al. Ramucirumab monotherapy for previously treated advanced gastric or gastro-oesophageal junction adenocarcinoma (REGARD): an international, randomised, multicentre, placebo-controlled, phase 3 trial. Lancet. 2014;383:31-9.

13. Wilke H, Muro K, Van Cutsem E, Oh S, Bodoky G, Shimada Y, et al. Ramucirumab plus paclitaxel versus placebo plus paclitaxel in patients with previously treated advanced gastric or gastrooesophageal junction adenocarcinoma (RAINBOW): a doubleblind, randomised phase 3 trial. Lancet Oncol. 2014;15:1224-35.

14. Di Bartolomeo M, Niger M, Laterza M, Vivaldi C, Giommoni E, Zaniboni A, et al. Safety and efficacy profile of ramucirumab alone or combined with paclitaxel in metastatic gastric cancer (MGC): a real-life overview of compassionate-use named patients (pts)(RAMoss study). Ann Oncol. 2016;27:636P.

15. Fuchs CS, Muro K, Tomasek J, Van Cutsem E, Cho JY, Oh S, et al. Prognostic factor analysis of overall survival in gastric cancer from two phase III studies of second-line ramucirumab
(REGARD and RAINBOW) using pooled patient data. J Gastric Cancer. 2017;17:132-44.

16. van Halsema E, van Hooft JE, Small AJ, Baron TH, García Cano $\mathrm{J}$, Cheon JH, et al. Perforation in colorectal stenting: a meta-analysis and a search for risk factors. Gastrointest Endosc. 2014;79:970.

17. Shojaei F. Anti-angiogenesis therapy in cancer: current challenges and future perspectives. Cancer Lett. 2012;320:130-7.

18. Fuchs CS, Tabernero J, Tomášek J, Chau I, Melichar B, Safran $\mathrm{H}$, et al. Biomarker analyses in REGARD gastric/GEJ carcinoma patients treated with VEGFR2-targeted antibody ramucirumab. Br J Cancer. 2016;115:974-82.

19. Tabernero J, Hozak R, Yoshino T, Cohn A, Obermannova R, Bodoky G, et al. Analysis of angiogenesis biomarkers for ramucirumab efficacy in patients with metastatic colorectal cancer from RAISE, a global, randomized, double-blind, Phase III Study. Ann Oncol. 2017. https://doi.org/10.1093/annonc/mdx767.

20. Jiang C, Liu S, He W, Zhang B, Xia L. The prognostic and predictive value of carbohydrate antigen 19-9 in metastatic colorectal cancer patients with first line bevacizumab containing chemotherapy. J Cancer. 2017;8:1410-6.

21. Yoshino T, Obermannová R, Bodoky G, Garcia-Carbonero R, Ciuleanu T, Portnoy DC, et al. Baseline carcinoembryonic antigen as a predictive factor of ramucirumab efficacy in RAISE, a secondline metastatic colorectal carcinoma phase III trial. Eur J Cancer. 2017;78:61-9.

\section{Affiliations}

\section{Minkyu Jung ${ }^{1} \cdot$ Min-Hee Ryu ${ }^{2}$ - Do Youn $\mathrm{Oh}^{3} \cdot$ Myounghee Kang ${ }^{4} \cdot$ Dae Young Zang ${ }^{5} \cdot$ In Gyu Hwang ${ }^{6}$. Keun-Wook Lee ${ }^{7}$ Ki Hyang Kim ${ }^{8}$. Byoung Yong Shim ${ }^{9}$. Eun Kee Song ${ }^{10}$. Sun Jin Sym ${ }^{11}$. Hye Sook Han ${ }^{12}$. Young Lee Park ${ }^{13}$. Jin Soo Kim ${ }^{14}$. Hyun Woo Lee ${ }^{15} \cdot$ Moon Hee Lee ${ }^{16}$. Dong-Hoe Koo ${ }^{17}$. Hong Suk Song ${ }^{18}$. Namsu Lee ${ }^{19}$. Sung Hyun Yang ${ }^{20}$. Dae Ro Choi ${ }^{21}$. Young Seon Hong ${ }^{22} \cdot K_{\text {Koung Eun Lee }}^{23}$. Chi Hoon Maeng ${ }^{24}$. Jin $\mathrm{Ho} \mathrm{Baek}^{25}$. Samyong $\mathrm{Kim}^{26}$. Yeul Hong $\mathrm{Kim}^{27}$. Sun Young Rha ${ }^{1}$ · Jae Yong Cho ${ }^{28}$ • Yoon-Koo Kang ${ }^{2}$}

1 Division of Medical Oncology, Yonsei Cancer Center, Department of Internal Medicine, Yonsei University College of Medicine, 50 Yonsei-Ro, Seodaemun-gu, Seoul, South Korea

2 Department of Oncology, Asan Medical Center, University of Ulsan College of Medicine, Seoul, South Korea

3 Department of Internal Medicine, Seoul National University Hospital, Cancer Research Institute, Seoul National University College of Medicine, Seoul, South Korea

4 Division of Hematology-Oncology, Department of Internal Medicine, Gyeongsang National University Changwon Hospital, Gyeongsang National University College of Medicine, Jinju, South Korea

5 Division of Hematology-Oncology, Department of Internal Medicine, Hallym University Medical Center, Hallym University College of Medicine, Anyang, South Korea

6 Department of Internal Medicine, Chung-Ang University College of Medicine, Seoul, South Korea

7 Department of Internal Medicine, Seoul National University Bundang Hospital, Seoul National University College of Medicine, Seongnam, South Korea

8 Division of Hematology-Oncology, Department of Internal Medicine, Inje University Busan Paik Hospital, Inje University College of Medicine, Busan, South Korea
9 Department of Medical Oncology, St. Vincent's Hospital, College of Medicine, The Catholic University of Korea, Suwon, South Korea

10 Department of Internal Medicine, Chonbuk National University Hospital, Chonbuk National University Medical School, Jeonju, South Korea

11 Department of Internal Medicine, Gachon University Gil Medical Center, Gachon University College of Medicine, Incheon, South Korea

12 Department of Internal Medicine, Chungbuk National University Hospital, Chungbuk National University College of Medicine, Cheongju, South Korea

13 Center for Gastric Cancer, Research Institute and Hospital, National Cancer Center, Goyang, Gyeonggi, South Korea

14 Department of Internal Medicine, Seoul National University Boramae Medical Center, Seoul, South Korea

15 Department of Hematology-Oncology, Ajou University School of Medicine, Suwon, South Korea

16 Division of Hematology-Oncology, Inha University Hospital and College of Medicine, Incheon, South Korea

17 Department of Internal Medicine, Kangbuk Samsung Hospital, Sungkyunkwan University School of Medicine, Seoul, South Korea 
18 Department of Internal Medicine, Dongsan Medical Center, Keimyung University, Daegu, South Korea

19 Department of Internal Medicine, Soonchunhyang University Hospital, Soonchunhyang University College of Medicine, Seoul, South Korea

20 Division of Hematology and Oncology, Department of Internal Medicine, Korea Cancer Center Hospital Korea, Korea Institute of Radiological and Medical Sciences, Seoul, South Korea

21 Department of Internal Medicine, Hallym University Medical Center, Hallym University College of Medicine, Chuncheon, South Korea

22 Division of Oncology, Department of Internal Medicine, Seoul St. Mary's Hospital, College of Medicine, The Catholic University of Korea, Seoul, South Korea

23 Division of Hematology-Oncology, Department of Internal Medicine, Ewha Womans University School of Medicine, Seoul, South Korea
24 Division of Hematology-Oncology, Department of Internal Medicine, Kyung Hee University College of Medicine, Seoul, South Korea

25 Department of Hematology and Oncology, Ulsan University Hospital, University of Ulsan College of Medicine, Ulsan, South Korea

26 Department of Internal Medicine, Chungnam National University College of Medicine, Daejeon, South Korea

27 Department of Internal Medicine, Anam Hospital, Korea University College of Medicine, Seoul, South Korea

28 Division of Medical Oncology, Gangnam Severance Hospital, Department of Internal Medicine, Yonsei University College of Medicine, 712 Eonjuro, Gangnam-gu, Seoul 06237, South Korea 